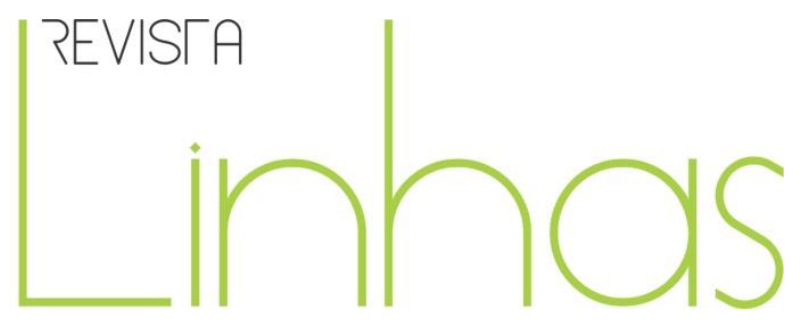

\title{
América Latina. La escuela sin territorio
}

\section{Resumen}

Este Artículo oficia como introducción al Dossier: Educación y Pandemia en América Latina. Realiza una presentación general de la problemática atendiendo a distintos aspectos. Por un lado, revisa y sistematiza información producida en 2020 respecto del cierre de las escuelas en los distintos países. Por otro lado, analiza algunos aspectos que se consideran condicionantes del desarrollo de estrategias y actividades de educación a distancia realizadas a través de distintos medios: la situación de conectividad y el acceso a las tecnologías digitales; la participación de estudiantes, docentes y miembros de la comunidad educativa en dinámicas de usos de tecnologías; la incidencia de los modelos de alfabetización vigentes, entre otros. Finalmente, reflexiona sobre las implicancias del traslado de las rutinas y actividades escolares al seno del hogar.

Palabras clave: Escuela. América Latina. Pandemia.

\section{Roxana Cabello}

Universidad Nacional de General

Sarmiento - Argentina rcabello@campus.ungs.edu.ar

\section{Para citar este artigo:}

CABELLO, Roxana. América Latina. La escuela sin territorio. Revista Linhas. Florianópolis, v. 22, n. 48, p. 4161, jan./abr. 2021. 


\section{Latin America. School América Latina. A escola sem without territory território}

\begin{abstract}
This Article serves as an introduction to the Dossier: Education and Pandemic in Latin America. It makes a general presentation of the problem taking into account different aspects. On the one hand, it reviews and systematizes information produced in 2020 regarding the closure of schools in the different countries. On the other hand, it analyzes some aspects that are considered conditioning factors for the development of distance education strategies and activities carried out through different means: the situation of connectivity and access to digital technologies; the participation of students, teachers and members of the educational community in dynamics of use of technologies; the incidence of current literacy models, among others. Finally, it reflects on the implications of moving school routines and activities within the home.
\end{abstract}

Keywords: School. Latin America. Pandemic.

\begin{abstract}
Resumo
Este artigo serve como uma introdução ao Dossiê: Educação e Pandemia na América Latina. Faz uma apresentação geral do problema levando em consideração diferentes aspectos. Por um lado, analisa e sistematiza a informação produzida em 2020 sobre o fechamento de escolas nos diferentes países. Por outro lado, analisa alguns aspectos considerados condicionantes para o desenvolvimento de estratégias e atividades de educação a distância realizadas por diferentes meios: a situação da conectividade e do acesso às tecnologias digitais; a participação de alunos, professores e membros da comunidade educacional na dinâmica de uso da tecnologia; a incidência dos modelos atuais de alfabetização, entre outros. Finalmente, reflete sobre as implicações da mudança de rotinas e atividades escolares dentro de casa.
\end{abstract}

Palavras-chave: Escola. América Latina. Pandemia. 
La canción Todo un palo (1988) es considerada como uno de los himnos del rock argentino y trascendió fronteras hacia el resto de América Latina. La primera línea de esa canción: "el futuro llegó hace rato..." (Un baión para el ojo idiota, 1988), se ha referido insistentemente en el último año para expresar las consecuencias que la situación de pandemia por Covid-19 ha producido sobre distintos aspectos de la vida cotidiana, sobre todo, en el ámbito de la educación.

Si decimos que el futuro llegó hace rato, desactivamos en parte el efecto sorpresa. La pregunta sobre qué nos depara el futuro, pareciera estar respondida y entonces las incertidumbres, pero también las expectativas y las promesas que conlleva quedan en suspenso. La frase “el futuro llegó hace rato" también puede imponer la idea de que existe un solo futuro, este que llegó. Pero cuando irrumpe la pandemia, afecta todos los futuros posibles.

En la primera mitad del siglo XIX se desarrolló la literatura de ciencia ficción, un nuevo género que buscaba, en principio, anticipar la manera en que el progreso cambiaría el mundo. La idea de que el futuro llegó cobraba vida en esos relatos que transmitían mayormente (aunque no siempre) una visión positiva del futuro, que confiaba en las posibilidades de las tecnologías para mejorar la vida de las personas. Ya en el siglo XX, las historias de ciencia ficción se hicieron eco del desencanto que desencadenaron las guerras y las revoluciones fallidas, el crecimiento de la pobreza y otros males de alguna manera relacionados con el avance tecnológico, y construyeron una visión distópica. Mundos post apocalípticos resultantes de una tecnología al servicio del poder deshumanizado marcaron una senda a la que se sumaron a lo largo del siglo otras preocupaciones como la destrucción del ambiente, el individualismo, la distancia entre ricos y pobres, y la pérdida de la libertad. En sus distintos lenguajes y formatos, la ciencia ficción demostró desde sus orígenes una enorme capacidad de observación (e información) sobre distintos aspectos de la vida social y cultural (incluidas la ciencia y la tecnología) y se dedicó a imaginar derivas posibles en escenarios futuros. Muchas de esas imaginaciones resultaron, con el tiempo, efectivas anticipaciones. 
Los virus y enfermedades inspiraron novelas: La peste escarlata (LONDON, 1912); Soy leyenda (MATHESON, 1954); Némesis (ROTH, 2010); Pandemia (THILLIEZ, 2015), entre otras, y también series y películas. La película Contagion (SODERBERGH, 2011) es quizá la narración que más directamente puede relacionarse con la situación que estamos atravesando a partir de la pandemia causada por el coronavirus SARS-CoV-2. Basada en la pandemia de Gripe A (H1N1), la historia problematiza varios de los aspectos que fueron convirtiéndose en componentes de nuestra cotidianeidad diez años después: la identificación de Oriente como la región de origen del virus; las condiciones de su globalización; las proyecciones sobre las consecuencias posibles del confinamiento de la población; el rol de los medios de comunicación; la expectativa sobre la producción de vacunas y sus implicancias políticas, científicas y económicas, entre otros. Varios años antes el film Outbreak (PETERSEN, 1995) mostraba una situación de cuarentena de la población frente a la amenaza de un virus similar al Ébola y seguramente pueden reconocerse otros antecedentes y variaciones como 28 days later (BOYLE, 2002) o la serie Helix (PORSANDEH, 2014).

Es posible que la situación de pandemia global actual, además de las graves consecuencias que acarrea en la vida real, imponga nuevos desafíos a la ciencia ficción porque parece que quedara ya poco margen para la anticipación ficcional.

En el caso de la educación, da la impresión de que las condiciones que impuso la pandemia produjeron una aceleración y una contracción de los tiempos. Esto es un rasgo que estamos habituados a analizar en relación con las tecnologías digitales y telemáticas a las que esta característica se les atribuye como efecto directo. Sin embargo, en este nuevo contexto observamos una suerte de efecto inverso: como consecuencia de este fenómeno de aceleración generado por la pandemia, se habría producido una tecnologización de los procesos educativos y desde un punto de vista podría decirse que terminó la espera que está siempre asociada a la idea de futuro.

Después de décadas de prédica respecto de que en la cultura contemporánea los aprendizajes se producen más allá de los muros de la escuela, este futuro completamente imprevisto y sólo imaginado en la ficción, arrasa literalmente con esos muros y pone to do en el marco del hogar. Esto da lugar a una serie de inestabilidades que están siendo analizadas por distintos actores de la comunidad educativa en reuniones académicas, en 
ámbitos gremiales, en intercambios entre docentes, que enfocan, por ejemplo, los modos como se modifican las relaciones entre estudiantes, padres o responsables y la escuela, tanto docentes como gestores escolares (SARTORI, 2020).

La integración de tecnologías (que sería el indicador de futuro) aparece como un puente (en mayor o en menor medida improvisado) entre esa escuela desterritorializada y ese hogar devenido, de manera abrupta, lugar de aprendizaje y tareas escolares.

\section{Escuelas cerradas}

Revisamos en este apartado la situación en la que se encuentran las escuelas en América Latina, considerando especialmente datos producidos por agencias internacionales que consideraremos aquí como primera síntesis, ya que permite visualizar un panorama general. De acuerdo con un informe de UNESCO actualizado en septiembre de 2020, la mayoría de los países de la región tenía las escuelas cerradas y a esa altura del año se anunciaba que se estaban preparando reaperturas (en ciertos casos, parciales), sobre todo en algunos países del área caribeña (UNESCO, 2020). El contexto impuso la necesidad de producir distintos tipos de respuestas educativas nacionales, incluyendo materiales didácticos, estrategias de capacitación y acompañamiento para docentes y/o familias. Según estimaciones del BID, para mediados de marzo había casi ciento sesenta millones de estudiantes en la región (veinte millones solo del nivel preescolar) que no estaban concurriendo a las escuelas (BID, 2020). En los distintos países fueron tomándose decisiones e implementando medidas y estrategias diferentes (ROIG, 2020): presencialidad con cupo reducido (en el caso de Colombia, en las escuelas privadas); implementación de protocolos sanitarios específicos (Chile), reapertura parcial en escuelas rurales (Ecuador, Perú); clases a distancia a través de medios de comunicación como radio y TV (México).

Sólo 3 países de la región regresaron de alguna manera a las clases presenciales durante 2020: Cuba, Nicaragua y Uruguay. En Cuba se anunciaba a principios de septiembre que las actividades presenciales se retomaban (con protocolos estrictos) en el $87.5 \%$ de los municipios del país, en los establecimientos de educación general (desde la enseñanza elemental hasta el bachillerato) y en las universidades de 9 de las 15 provincias. El ministro 
de Educación Superior, José Ramón Saborido, declaró: “Donde sea posible, [... ], debemos comenzar el curso escolar, no tenemos el derecho de postergarlo, pues el país, los territorios, necesitan la formación de los profesionales, que es la tarea de la enseñanza superior" (AGENCIA PRENSA LATINA, 2020). El 12 de noviembre UNICEF anunciaba que alrededor de trescientos doce mil estudiantes de distintos niveles asistían a las aulas en La Habana, luego de más de siete meses de cierre por el problema de Covid-19.

Uruguay, por su parte, retomó gradualmente el sistema presencial no obligatorio a partir de junio. El nivel medio culminó el ciclo lectivo mientras que en el resto de los niveles volvió a interrumpirse la presencialidad a principios de diciembre como prevención por el aumento de casos de coronavirus en el país (PLANTEAN..., 2020).

Las decisiones de los gobiernos de los distintos países respecto de mantener las clases presenciales estuvieron condicionadas por las opiniones y reclamos de los distintos sectores integrantes de la comunidad educativa. En el caso de Nicaragua, la Federación Coordinadora Nicaragüense de ONGs que Trabajan con la Niñez y la Adolescencia del país, denunció en el mes de junio la falta de acciones del gobierno para reducir los riesgos de contagios por Covid-19 a los más de un millón ochocientos mil estudiantes, docentes y personal administrativo del sistema de educación y sus familias (REDCLADE.ORG, 2020). La Organización Mundial de la Salud (OMS) y la Organización Panamericana de la Salud (OPS) expresaron preocupación sobre Nicaragua, ya que no solamente no suspendió las clases presenciales en medio de la pandemia en 2020, sino que además casi no estableció restricciones. Si bien no hay disponibilidad de datos oficiales sobre el impacto de la pandemia en las escuelas, la Unidad Sindical Magisterial informa que durante 2020 al menos cuarenta y cuatro docentes fallecieron con síntomas de Covid-19 (NEWSROOM INFOBAE, 2021) y la Asociación Médica de Nicaragua alerta sobre las posibles consecuencias de mantener las escuelas abiertas respecto de la propagación de la enfermedad. De todos modos, el Ministerio de Educación de este país anunció que el 1 de febrero de 2021 se producirá el retorno a las aulas con el inicio del nuevo ciclo lectivo.

Hasta tanto no se cuente con estudios específicos y confiables que demuestren la incidencia de la apertura de las aulas en el aumento de los contagios, las decisiones que tomen los ministerios de educación de las naciones latinoamericanas podrán tener en 
cuenta las recomendaciones básicas que realizan las organizaciones internacionales dedicadas al cuidado de la salud. La OMS recomienda que la enseñanza presencial se realice en ambientes bien ventilados, con la amplitud suficiente para mantener la distancia social y con disponibilidad de servicios básicos (agua potable y sanitarios) para promover y desarrollar prácticas de higiene como el lavado de manos.

Un factor fundamental para retomar las clases presenciales es crear las condiciones para mantener la distancia social. La OMS recomienda que la distancia mínima entre los asientos de los alumnos sea de un metro a cada lado. Esto requiere un aula con una superficie de 2,5 metros cuadros por alumno. Una indagación producida en once países de América Latina y el Caribe estima que el promedio a nivel nacional de metros cuadrados disponibles por alumno en el aula oscila entre 1,5 -en Argentina, Chile y Guatemala- y 1,8 -en Barbados y Colombia- (MARIS; ESTRADA, 2020). Se impone entonces diseñar estrategias alternativas como reducir el número de estudiantes que participan en cada clase, lo cual puede realizarse abriendo de manera escalonada o aumentando la cantidad de actividades al aire libre (esto requiere atender a cuestiones de logística, por ejemplo, vinculadas con el transporte escolar).

En cuanto a la disponibilidad de servicios básicos, Maris y Estrada (2020) analizan los datos de TERCE (2013) y concluyen en que en algunos países de la región (Argentina, Colombia, México, Panamá y Perú) menos de la mitad de los colegios que atienden a los estudiantes más pobres cuentan con la infraestructura básica necesaria como para garantizar las condiciones sanitarias requeridas. El acceso al agua potable y los sanitarios en buen estado está más garantizado en las escuelas a las que concurren estudiantes de nivel socioeconómico más alto que en las que reciben a los estudiantes de nivel socio económico bajo.

Los distintos tipos de brechas y desigualdades que aquejan a la niñez y la adolescencia en la región, pueden profundizarse a partir de la emergencia que estamos atravesando desde principios de 2020.

Los chicos y las chicas que están cursando el primer ciclo de la escuela primaria, posiblemente se encuentren entre la población escolar más afectada por esta situación que 
mantiene las escuelas cerradas y refuerza las iniquidadesii. El aprendizaje del lenguaje escrito es un proceso de apropiación complejo, de alto compromiso cognitivo, que requiere tiempo y atención; se adquiere en una situación de comunicación y diálogo con otros y exige un trabajo consciente y analítico de los elementos que constituyen el lenguaje. Necesita un acompañamiento e intervención pedagógica.

En el Estudio sobre procesos de apropiación de tecnologías entre niños y niñas de seis a ocho años que realizamos en el Programa Usos de Medios Interactivos (IDH-UNGS) entre 2017 y 2020, constatamos que buena parte de la población infantil de ese tramo de edad con la que hemos estado trabajando, que proviene de familias de menores recursos del área periurbana de Buenos Aires, comienza el primer año y atraviesa en general el primer ciclo de la escuela, teniendo pocas y poco variadas experiencias con el lenguaje escrito en el contexto familiar, escaso conocimiento sobre el cual comenzar a construir. Requieren entonces más y mejor acompañamiento y orientación. Efectivamente, las diferentes experiencias con la lengua escrita inciden en los conocimientos con los que los niños ingresan a la escuela (MANRIQUE; GRANATO, 1995; PIACENTE; TALOU; RODRIGO, 1990), y remiten a la necesidad tanto de intervenciones tempranas como de intervenciones sistemáticas en los primeros grados.

Sin embargo, este problema no afecta únicamente a niños y niñas de esas edades, sino que ya antes de la pandemia la región enfrentaba una crisis educativa, con muy altos grados de pobreza de aprendizaje en los distintos niveles educativos e inequidades persistentes. Según di Gropello (LA EDUCACIÓN..., 2020), alrededor del 50\% de los estudiantes no podía leer de manera apropiada a la edad de 10 años. Especialistas y analistas de agencias internacionales coinciden en señalar que es posible que como consecuencia de la situación de la escolaridad 2020, se profundicen las pérdidas educativas, se agudicen las desigualdades y aumenten las deserciones escolares.

\section{Migración digital apremiada}

La urgente necesidad de prevenir los contagios de Covid-19 impuso la instrumentación acelerada de la enseñanza a distancia en distintas modalidades (en 
algunos casos, Ilamada virtual) y con diferentes alcances en los países de América Latina. A pesar de las políticas denominadas de inclusión digital que se implementaron en la región, podría decirse que las condiciones de partida que tuvo este proceso en los inicios de 2020 no fueron las mejores, si se consideran distintos aspectos. Un estudio publicado en Bolivia en 2019 sintetiza la situación que se verifica en ese país y que, tal como veremos, comparte rasgos con otros de la región:

Desde el lado de la educación, aunque es evidente un mayor uso de computadoras y de internet en los ámbitos educativos, persisten vacíos en el área, relacionados a la calidad de la conexión a internet, falta de equipamiento, escasez de habilidades en el uso de TIC, brechas entre localidades y niveles socioeconómicos, y la preferencia por la educación presencial. Este último dato está asociado principalmente a una mayor confianza hacia la educación presencial, a la cual se le atribuyen cualidades como la seriedad. (AGETIC; UNFPA, 2019, p. 102)

Retomemos algunos de los aspectos allí señalados que condicionan la migración digital (CABELLO, 2013) de los procesos educativos. En primer lugar, la cuestión de la conectividad.

La conexión a internet se reveló como un requerimiento indispensable para la participación de estudiantes de todos los niveles educativos en las propuestas pedagógicas que se desarrollaron durante 2020. Las condiciones de conectividad que caracterizan a los países de la región demuestran concentración en los centros urbanos, diferencias de acceso en los distintos quintiles de la sociedad y brechas que se profundizan en especial en relación con la disponibilidad de banda ancha en los hogares (lo cual repercute en la calidad y posibilidades de uso). Por otra parte, en muchos hogares de menores ingresos la conexión se realiza a través de dispositivos móviles (mayormente teléfonos celulares).

María Elena Giraldo-Ramírez y Gloria María Álvarez Cadavid analizan en un artículo que forma parte de este dossier, los datos del censo nacional de Colombia que indican que en 2018 ese país tenía un 43\% de conectividad en los hogares, concentrado sobre todo en las ciudades y en los estratos medios y alto. Las autoras sostienen que en 2018 el $63 \%$ de los bachilleres de Colombia no tenía acceso a internet desde sus hogares (GIRALDO RAMíREZ; ÁLVAREZ CADAVID, 2021). 
Uruguay, un país en donde las políticas públicas de inclusión digital en la Sociedad de la Información y el Conocimiento tienen una de las historias más largas y sostenidas de la región, muestra un panorama un tanto mejor. Como sostienen María Julia Morales González y Mauricio Olivera en el artículo que se publica en este dossier, la brecha entre los hogares del quintil 1 y los del quintil 5 en relación con la conexión a Internet no alcanza el diez por ciento. Sin embargo, la distancia se hace más significativa cuando se toma como parámetro la conexión de banda ancha fija. En ese caso, la brecha llega al treinta y cinco por ciento, y una distancia similar se observa en relación con la tenencia de computadora en el hogar (MORALES GONZÁLEZ; OLIVERA, 2021).

Como se observa, aun teniendo en cuenta esta ventaja respecto de otros países, el análisis pone de manifiesto las diferencias de acceso que todavía persisten si se consideran los distintos sectores económico sociales. Además, el artículo da cuenta de que el factor de conectividad no es el único que interviene en este proceso de migración digital que busca garantizar la continuidad de los procesos de enseñanza y aprendizaje. Enfocaremos algunos de los otros factores que están operando.

Sin dudas la cuestión del acceso y los_usos de las tecnologías digitales por parte de estudiantes y docentes es central. Como hemos visto, la conectividad básica a internet es un problema para los hogares de los países más pobres y de las zonas rurales. Pero, tal como señala un informe de Unicef (2017) las divisiones de "segundo nivel”, que trascienden el acceso y se relacionan con los usos que realizan los niños, niñas y adolescentes, tienen cada vez más relevancia para cerrar la brecha digital.

En el caso de la educación en esta situación de emergencia, el problema del acceso continúa siendo relevante porque se relaciona con las oportunidades que tienen las personas en edad escolar y de estudios superiores de participar en el conjunto de opciones que van produciendo las instituciones educativas. Por ejemplo, el Banco Interamericano de Desarrollo (BID, 2020) informa que sólo el $64 \%$ de los estudiantes de la región tiene una computadora en el hogar, lo cual dificulta el acceso a las plataformas digitales de aprendizaje de una porción muy importante (ROIG, 2020). 
Un examen de datos producidos por la Encuesta Permanente de Hogares en Argentina, permite comprobar que 5 de cada 10 personas dentro de la población infantil de 6 a 8 años del conurbano de Buenos Aires, que están bajo la línea de pobreza, tiene un vínculo restringido o directamente no acceden ni utilizan computadora, internet y telefonía celular (MOYANO, 2020). Pero si se considera la población escolar general, casi un $60 \%$ de los estudiantes utilizaron sólo teléfono celular para sus tareas escolares durante la pandemia 2020, dispositivos que en un casi $70 \%$ no eran propios, sino que pertenecían a otro integrante de la familia (MORALES, 2021).

Más allá del acceso, hemos identificado que un factor que resulta fundamental en relación con los tipos de vínculos que se construyen con las tecnologías digitales es la participación en determinadas dinámicas de usos (CABELLO, 2019). Los usos y competencias de las personas adultas con las que se relacionan en el hogar y en la escuela ofician como modelos, orientación para los usos y formación de competencias, y usina de imaginaciones sobre las tecnologías por parte de niños, niñas y adolescentes. La participación en estas dinámicas de usos genera condiciones para la apropiación de tecnologías entendida como aprendizaje social que se realiza en actividades y que se interioriza a la manera de vivencias, atravesadas en este caso por aspectos emocionalesafectivos.

A través de análisis cualitativos en hogares y en escuelas, hemos visto que niños y niñas en edad escolar participan en dinámicas de usos de tecnologías en el hogar predominantemente orientadas hacia el entretenimiento y a partir de dispositivos móviles compartidos. Este tipo de dinámicas involucra unas modalidades de significación de las tecnologías y sus posibilidades por parte de los niños y las niñas, que requieren reacomodamientos si se busca promover usos vinculados con prácticas de aprendizaje escolar.

Un estudio de CEPAL y UNESCO (2020) explica que en 2018 la mayoría de los estudiantes de 15 años de edad de los siete países de la región que participaron en la prueba PISA realizaban algunas actividades relacionadas con el trabajo escolar usando alguna tecnología: comunicarse con los docentes, hacer tareas y buscar información en Internet o hacer seguimiento de contenidos de estudio. Sin embargo, sostiene también que no todos 
los estudiantes tienen esa experiencia previa e identifica factores de diferenciación. Por un lado, aclara que las diferencias por nivel socioeconómico y cultural de los estudiantes condicionan la realización de todas las actividades de manera tal que cuando mayor es el nivel socioeconómico y cultural, mayor es la proporción de estudiantes que tienen experiencia en la actividad. Por otro lado, señala que la realización de actividades en línea también difiere según la edad y aumenta de manera importante en la adolescencia. Dice el informe:

\begin{abstract}
Es en la adolescencia cuando comienza el acercamiento a Internet a través de actividades relacionadas con la socialización y el entretenimiento, por lo que es probable que los niños y niñas de primaria estén en desventaja para asumir esta continuación de estudios de manera virtual a través de Internet. (CEPAL; UNESCO, 2020, p. 7)
\end{abstract}

Otro tanto sucede con los y las docentes. Las características del tipo de formación que han recibido y de los modelos pedagógicos y didácticos en los que se enmarcan sus prácticas laborales (así como institucionales) suelen estar ajenos a las dinámicas de usos de las tecnologías que más promueven la formación de competencias y el diseño e implementación de estrategias de digitalización de las propuestas educativas. La situación de pandemia y las consecuencias directas que ha tenido sobre la organización del ciclo lectivo, ha impuesto una integración forzada a una dinámica de usos muchas veces improvisada o cuanto menos, desconocida. Algunas referencias pueden leerse en otros artículos incluidos en este mismo dossier. Por ejemplo, Susana Morales, de Argentina, observa que los y las docentes tuvieron que aprender e imaginar usos de las tecnologías que les permitieran continuar con la enseñanza más allá de la presencialidad, y señala el contexto complejo de precarización del trabajo docente y del acceso a las tecnologías en América Latina (MORALES, 2021). Nuevos aprendizajes e imaginaciones que se asocian con otra idea que se instala en varios análisis: la de la reinvención. Como dicen Adriana Barroso de Azevedo, Elaine Gomes Vilela y Fabiana Moreira Gaviolli, de Brasil, refiriéndose al caso de la educación superior: con la propagación de la pandemia los profesores pasaron por un proceso de reinvención y también todas las instituciones de enseñanza superior pasan por un proceso de transición, buscando alternativas para dar continuidad a los procesos de enseñanza y de aprendizaje (AZEVEDO; VILELA; GAVIOLLI, 2021). 
La reinvención puede adoptar distintas formas y vivenciarse con diversos grados de conciencia y/o planificación. Roberto Canales Reyes observa, en el caso de Chile, que los y las docentes trabajan en forma coordinada para generar estrategias pedagógicas conjuntas, apelando a la autoafirmación y el autoaprendizaje, pero también al apoyo de profesionales psicosociales y de programas oficiales como el de Integración Escolar (CANALES REYES, 2021).

Respecto de los distintos tipos de estrategias, en nuestro estudio cualitativo realizado en escuelas primarias del conurbano de Buenos Aires recogimos la impresión de madres y padres de niños y niñas de primer ciclo que entendían que más que integración de tecnologías, las y los docentes hacen esfuerzos por acomodarse a la situación: muchas veces no tienen computadora o buena conectividad y sus usos predominantes son también de tipo recreativo y comunicacional. Puede verse que han logrado aprovechar los usos con los que se sienten más familiarizados/as: por ejemplo: usan bastante los grupos de mensajería instantánea (tipo WhatsApp) para enviar consignas de trabajo y elaboran videos breves con el celular, que funcionan como tutoriales de esas consignas cuyos destinatarios principales son los niños y las niñas, pero que mayormente requieren la mediación de personas adultas para facilitar la recepción (tanto técnica como semántica).

No solo los y las docentes, sino también las autoridades de los centros educativos y el propio funcionamiento institucional, tuvieron que adecuarse a estas condiciones de desterritorialización de las prácticas educativas. Muchas veces, esos procesos se produjeron según las posibilidades de cada institución y de sus comunidades educativas, con independencia de las orientaciones de política educativa que no siempre llegaron a tiempo. Así comentan Morales González y Olivera, para el caso de la escuela media en Uruguay, que observan que docentes y autoridades tuvieron que coordinar estrategias conjuntas para acelerar los procesos de formación de competencias y sobrellevar las dificultades técnicas y pedagógicas. Señalan que a pesar de que se ofreció formación y material a los docentes desde el Plan Ceibal, la situación de urgencia y desorden que se experimentó en las escuelas produjo demoras en la llegada de ese acompañamiento (MORALES GONZALEZ; OLIVERA, 2021). 
Una de las dimensiones que se ponen de relieve, que no habíamos considerado hasta el momento, es la de las oportunidades que esta nueva coyuntura ofrece en relación con la transformación educativa. Por ejemplo, el avance en el trabajo colaborativo entre integrantes del personal docente, y la formación de competencias digitales; dos aspectos centrales y poco desarrollados.

Consideramos que uno de los factores que repercute en esa situación es el tipo de modelo de alfabetización que se adopta en las instituciones en particular y que se promueve desde las políticas públicas en general. Los chicos y las chicas aprenden a construir competencias comunicativas complejas mayormente en el ámbito escolar. Nuestro estudio nos ha permitido constatar que, en muchas escuelas del conurbano de Buenos Aires, al igual que como sucede en otras regiones periurbanas de América Latina, la atención está puesta en la enseñanza de la lectura y la escritura, más que en el desarrollo de modelos de alfabetizaciones múltiples o de la llamada alfabetización mediática digital, que se define como "[... ] la capacidad de obtener acceso a comunicaciones, así como de comprenderlas y crearlas en una variedad de contextos" (BUCKINGHAM, 2008, p. 192). A diferencia de las definiciones funcionales de tipo instrumental, se reconoce en este tipo de enfoques, una dimensión crítica de la alfabetización que supone la capacidad de analizar, evaluar y reflexionar sobre y con las tecnologías. La reflexión sobre qué significa alfabetizar en el mundo contemporáneo, qué rol juega el lenguaje escrito y qué sentido tiene promover procesos de apropiación de tecnologías (sus lenguajes y productos) en la comunidad educativa en relación con esos propósitos, está menos desarrollada de lo que hubiera sido deseable para favorecer mejores condiciones para docentes y estudiantes.

\section{La escuela en casa}

La escuela no solamente es el lugar donde se producen aprendizajes escolares, sino donde entramos en contacto con otras personas que provienen de hogares-mundos distintos y donde algunas desigualdades quedan en parte neutralizadas. Sobre todo, en la escuela pública, que es en donde se juega la equidad en lo que respecta al acceso a la información y el conocimiento que debería permitirnos ser parte activa de las relaciones 
sociales y la producción de la cultura. La escuela (en general) es también el lugar en donde desarrollamos afectos y apuntalamos nuestra subjetividad en distintas etapas de la vida; donde podemos ser nosotros/as mismos (cada uno/a de los que somos en cada etapa) en relación con pares y con personas adultas, normas y modelos ajenos a los familiares.

De manera que la escuela es un ámbito colectivo completamente diferente del hogar-mundo, privado, singular, en donde imperan otras reglas y relaciones de poder, en donde se definen otros roles y estilos de comunicación.

La inserción de rutinas, prácticas de aprendizaje y actividades escolares en el hogar estuvo históricamente asociada con la llamada "tarea para el hogar": trabajo complementario del realizado en la escuela, que muchas veces requiere el acompañamiento de personas adultas u otros integrantes. Sin embargo, el contexto de pandemia por Covid-19 impuso la migración digital o, al menos, la distanciación de la educación en los distintos niveles, y puso al hogar en el centro de la escena del proceso de enseñanza y aprendizaje. La escuela se desterritorializa en parte (no del todo, porque se cierra un poco sobre sí misma, conservando su territorio frente a la expectativa de un futuro incierto). Pero en el contexto de aislamiento o distanciamiento social preventivo, esa desterritorialización se apoya en flujos de comunicación que requieren unas condiciones de recepción que se hacen más eficaces cuanto más territorializadas están. Aún con la preminencia de los dispositivos móviles, la casa (de un modo u otro) ofrece unas condiciones de estabilidad que favorecen la comprensión de las consignas de trabajo por parte de las personas que orientan a los y las estudiantes. Consignas para resolver unas actividades que, además, están diseñadas en muchísimos casos para solucionar con lápiz y papel.

El hogar deviene entonces el territorio en donde se incluyen las tareas escolares en una dinámica de usos de tecnologías digitales que no las tenía previstas. Distintos/as integrantes se ven en la situación de aprender a usar para ayudar a usar las tecnologías que permiten recibir (en algunos casos, resolver) y enviar las tareas realizadas; además de orientar en la resolución general de las tareas que ya no son complementarias, sino centrales dentro de un proceso en el cual está involucrada la promoción y permanencia de niños, niñas y adolescentes en el sistema educativo. Cada estudiante trae su escuela, sus 
docentes, sus tareas, sus demandas, al mundo privado del hogar en donde se produce una superposición en la que participan también prácticas laborales y domésticas. Además de las disputas por los espacios o los tiempos de uso de las tecnologías, entre otros aspectos, un nuevo rol desconocido comienza a tomar forma improvisadamente: madres (u otras personas) haciendo de docentes, sin querer, sin saber, sin sentirse en condiciones, muchas veces sin legitimidad.

Por otra parte, no todas las viviendas ni todos los hogares son iguales. De acuerdo con un informe de CEPAL y UNESCO, en América Latina

[... ] más de 80 millones de niñas, niños y adolescentes de zonas urbanas enfrentan algún tipo de privación en sus condiciones habitacionales y unos 18 millones residen en hogares con precariedad habitacional grave. [... ] el hacinamiento impide contar con un espacio adecuado para estudiar y descansar, lo que repercute en el desarrollo cognitivo en la infancia y las trayectorias laborales y de bienestar en la adultez, a la vez que favorecen una mayor propensión a situaciones de abuso. (CEPAL; UNESCO, 2020, p. 14)

En esas condiciones, difícilmente el hogar pueda funcionar como algo parecido a un salón de clase y tal vez menos personas estén en condiciones de asumir un rol cuasi docente.

Estamos atravesando un período difícil en el que las familias han visto modificadas sus rutinas y sus actividades y, en muchísimos casos, alteradas sus estrategias y fuentes de subsistencia. En ese contexto, se agrega no solamente el hecho de que se multiplica el número de personas en el hogar varias horas por día (porque hay quienes no están en la escuela o en el trabajo), sino el estrés que implica hacerse cargo de la actividad escolar. De manera que el acompañamiento de la escuela se torna crucial.

Como señalan Morales González y Olivera (2021), uno de los desafíos que afrontaron los centros educativos fue el de mantener las conexiones con las familias para no perder el vínculo con los estudiantes, y la pandemia puso en evidencia en qué situación estaban esas conexiones (condicionadas por desigualdades socioeconómicas, educativas y culturales) cuando se inició este proceso. Es fundamental que esas conexiones se refuercen. Siempre lo ha sido, pero en este contexto lo es aún más porque la escuela puede buscar la manera 
de continuar ofreciendo acompañamiento, contención y apoyo emocional. En esta nueva configuración que asume el ecosistema educomunicacional (SARTORI, 2021), la escuela irá ensayando nuevos roles y perfiles para sus integrantes y también puede incluir la dimensión socioemocional entre los aprendizajes (CEPAL; UNESCO, 2020).

Más que nunca es la propia escuela pública (como entramado vivo de relaciones) la que también necesita contención, para poder pensarse, consolidarse aprovechando los aprendizajes medio forzosos de esta etapa y fortalecer sus redes internas en un escenario en donde el riesgo de dispersión es muy importante y en el que, como hemos dicho, su vínculo con el hogar es más significativo que en otros tiempos.

\section{El dossier como comunidad de aprendizaje}

En el momento en el que estamos preparando este dossier, el estado actual de la educación y la relación entre la escuela y las familias es uno de los principales temas de la conversación social en los países de América Latina.

Para hacer un aporte desde el ámbito académico de la región, hemos priorizado seleccionar un conjunto de investigaciones que permiten recoger la perspectiva de los actores de diferentes niveles del sistema educativo en distintos países, con la intención de aportar sensaciones y evaluaciones respecto de los avances producidos, los requerimientos, las faltas, los aprendizajes. A partir de ese contacto estrecho con los lugares en donde se producen los encuentros y desencuentros educativos en el contexto de la pandemia por Covid-19, se presentan contribuciones y propuestas.

Los trabajos comparten la inquietud de intentar comprender y caracterizar en tiempo real la situación inédita en que se encuentra la educación en un conjunto de zonas de Latinoamérica, en un contexto también inédito. Enfocan varios temas y problemas, entre los que cuentan las condiciones en las que se encuentra el sistema educativo y su comunidad en el momento de inicio de la pandemia; el rol y alcance de las políticas públicas de inclusión digital; los desafíos para las instituciones educativas y sus comunidades; y las oportunidades que presenta la coyuntura respecto del desarrollo de propuestas educomunicativas con integración de tecnologías. 
Confiamos en que estos artículos ofrezcan una contribución a esa conversación a la que hacíamos referencia y al diseño e implementación de acciones que permitan capitalizar los aprendizajes que esta coyuntura tan particular nos está propiciando realizar.

\section{Referências}

AGENCIA PRENSA LATINA. Reinicia el curso escolar en Cuba con medidas especiales ante la COVID-19. Escambray, Sancti Spiritus, 1 sept. 2020. Disponible en:

http://www.escambray.cu/2020/reinicia-el-curso-escolar-en-cuba-con-precaucionesespeciales-ante-la-covid-19/ Acceso en: 10 de octubre de 2020.

AGETIC; UNFPA. Juventudes TIC: estudio sobre las TIC en adolescentes y jóvenes en Bolivia. La Paz: AGETIC; UNFPA, 2019.

AZEVEDO, Adriana Barroso de; VILELA, Elaine Gomes; GAVIOLLI, Fabiana Moreira. Educação remota no ensino superior em tempos de pandemia. Dossier Educación y Pandemia en América Latina. Revista Linhas, Florianópolis, v. 22, n. 48, 2021.

BID. ¿Volvemos a las aulas?: primeros pasos. [Buenos Aires], 16 nov. 2020. Disponible en https://blogs.iadb.org/desarrollo-infantil/es/volvemos-a-las-aulas-opinion/. Acceso en: 17 nov. 2020.

BUCKINGHAM, David. Más allá de la tecnología: aprendizaje infantil en la era de la cultura digital. Buenos Aires: Manantial, 2008. 
CABELLO, Roxana. Estrategias para el estudio de procesos de apropiación de tecnologías en la infancia. En: RIVOIR, Ana; MORALES, María (coords.). Tecnologías Digitales: miradas críticas de la apropiación en América Latina. Buenos Aires: CLACSO, 2019. p. 103-122.

CABELLO, Roxana. (coord.). Migraciones digitales: comunicación, educación y tecnologías digitales interactivas. Buenos Aires: UNGS, 2013.

CANALES REYES. Roberto. La educación como un derecho: Chile en tiempos de pandemia. Dossier Educación y Pandemia en América Latina. Revista Linhas, Florianópolis, v. 22, n. 48, feb. 2021.

CEPAL; UNESCO. La educación en tiempos de la pandemia de COVID-19. Santiago, ago. 2020. Disponible en:

https://repositorio.cepal.org/bitstream/handle/11362/45904/S2000510_es.pdf?sequence=1 \&isAllowed=y. Acceso en: 10 oct. 2020.

GIRALDO-RAMÍREZ, María Elena; ÁLVAREZ CADAVID, Gloria María. Educación en tiempos de pandemia: tensiones, evidencias y emergencias de la relación educación y tecnología. Dossier Educación y Pandemia en América Latina. Revista Linhas, Florianópolis, v. 22, n. 48, 2021.

LA EDUCACIÓN en américa latina enfrenta una crisis silenciosa, que con el tiempo se volverá estridente. Banco Mundial noticias. Washington D.C, 1 de junio de 2020.

Disponible https://www.bancomundial.org/es/news/feature/2020/06/01/covid19coronavirus-educacion-america-latina acceso 10 de diciembre de 2020.

MANRIQUE, Ana María; GRANATO, Luis. Discurso narrativo: algunos aspectos del desempeño lingüístico en niños de diferente procedencia social. Lenguas Modernas 22, Santiago, 137-166, 1995. Disponible en

https://revistas.uchile.cl/index.php/LM/article/view/45565/47628 Consultado el 3 de marzo de 2021.

MARIS, Lesbia; ESTRADA, Ricardo. ¿Están preparadas las escuelas de América Latina para un regreso seguro a clases? CAF - Banco de Desarrollo de América Latina, Caracas, 3 nov. 2020. Disponible en: https://www.caf.com/es/conocimiento/visiones/2020/11/estanpreparadas-las-escuelas-de-america-latina-para-un-regreso-seguro-a-clases/. Acceso en: 1 dic. 2020.

MORALES GONZÁLEZ, María Julia; OLIVERA, Mauricio. (Des)conexiones y pandemia: entre las políticas de educación pública remota de emergencia y las escuelas de enseñanza media en Uruguay. Revista Linhas, v. 22, n. 48. Florianópolis, 2021. 
MORALES, Susana. Argentina 2020: vicisitudes y dilemas de la educación pública en contextos de pandemia. Revista Linhas, Florianópolis, v. 22, n. 48, 2021. Dossier Educación y Pandemia en América Latina.

MOYANO, Renzo. Brecha social y brecha digital. Pobreza, clima educativo del hogar e inclusión digital en la población urbana de Argentina. Signo $\mathbf{Y}$

Pensamiento, Bogotá, v. 39, n. 77, 2020. Disponible en: https://revistas.javeriana.edu.co/index.php/signoypensamiento/article/view/32262 Acceso en: 2 enero 2021.

NEWSROOM INFOBAE. Nicaragua cita a clases presenciales a $\mathbf{1 . 7}$ millones de estudiantes. Infobae. Buenos Aires, 11 enero 2021. Disponible en: https://www.infobae.com/america/agencias/2021/01/11/nicaragua-cita-a-clasespresenciales-a-17-millones-de-estudiantes/ Acceso en: 11 enero 2021.

PIACENTE, Telma; TALOU, Carmen; RODRIGO, María. Piden Pan ... y algo más: un estudio sobre crecimiento y desarrollo infantil. Buenos Aires: UNICEF-SIGLO XXI, 1990.

PLANTEAN suspender las clases presenciales y actos de fin de curso por aumento de casos. El País, Montevideo, 1 dic. 2020. Disponible en: https://www.elpais.com.uy/informacion/educacion/plantean-suspender-clasespresenciales-actos-curso-aumento-casos.html. Acceso en: 1 dic. 2020.

REDCLADE.ORG. Nicaragua: organizaciones exigen la inmediata suspensión de las clases presenciales durante la pandemia. [S.I.], 8 jun. 2020. Disponible en: https://redclade.org/noticias/nicaragua-organizaciones-exigen-la-inmediata-suspensionde-las-clases-presenciales-durante-la-pandemia/. Acceso en: 10 de octubre de 2020.

ROIG, Catalina. ¿Cómo es la vuelta a las clases presenciales en América Latina? Chequeado, Buenos Aires, 29 nov. 2020. Disponible en: https://chequeado.com/elexplicador/como-es-la-vuelta-a-las-clases-presenciales-en-america-latina/. Acceso en: 1 dic. 2020.

SARTORI, Ademilde Silveira. Ecossistema educomunicativo: comunicação e aprendizagem em rede. Dossier Educación y Pandemia en América Latina. Revista Linhas, Florianópolis, v. 22, n. 48, 2021.

SARTORI, Ademilde Silveira. Pensando ecosistemas educomunicativos en tiempos de Covid19. En Technos Magazine Digital, Buenos Aires, n. 8, jul. 2020. Disponible en: http://technosmagazine.com.ar/8aprended.html Acceso en: 2 dic. 2020.

UNESCO. La educación en América Latina y el caribe ante el COVID-19. [S.I.], 20 sept. 2020. Disponible en: https://es.unesco.org/fieldoffice/santiago/covid-19-educationalc/respuestas. Acceso en: 24 oct. 2020. 
TERCE. Tercer estudio regional comparativo y explicativo. [S.I.]: UNESCO, 2013. Disponible en: https://es.unesco.org/fieldoffice/santiago/llece/TERCE2013. Acceso en: 24 oct. 2020.

UNICEF. Estado mundial de la infancia 2017. Niños en un mundo digital. [S.I.], dic. 2017. Disponible en: https://www.unicef.org/media/48611/file. Acceso en: 2 feb. 2018.

Recebido em: 13/02/2021 Aprovado em: 28/03/2021

Universidade do Estado de Santa Catarina - UDESC Programa de Pós-Graduação em Educação - PPGE Revista Linhas Volume 22 - Número 48 - Ano 2021 revistalinhas@gmail.com

\footnotetext{
' Un Baión para el ojo idiota. Intérprete: Patricio Rey y sus Redonditos de Ricota. Compositores: S. Bellinson e I. Solari. [S./.]: Del Cielito Argentina, 1988. 1CD.

ii Una primera versión de las observaciones que se presentan aquí al respecto, se comunicaron en el panel "La educación en tiempos de pandemia", del encuentro Las tecnologías digitales y la pandemia. Miradas desde las ciencias sociales: el futuro llegó hace rato, organizado por el Instituto de Investigación Gino Germani de la Universidad de Buenos Aires, Argentina, 27 al 30 de octubre de 2020.
} 